\title{
HOW NEUROIMAGING CAN HELP US TO VISUALISE AND QUANTIFY PAIN?
}

\section{Karolina Wartolowska}

Nuffield Division of Anaesthetics, Nuffield Department of Neurological Sciences, University of Oxford, Oxford, UK

\begin{abstract}
Pain is a complex and multidimensional experience, which is subjective for an individual and modulated by physiological and psychological factors. Therefore, it is difficult to quantify pain and there are no objective pain measures available at the moment. Neuroimaging provides an objective measure of changes in brain activity related to pain perception. In this review, we demonstrate that pain-related brain activation is complex and can be best studied as a dynamic network of interconnected regions. Finally, we use the placebo and nocebo effects to discuss the factors involved in modulation of pain experience.
\end{abstract}




\section{Neuroimaging techniques}

Neuroimaging is a valuable tool for visualising pain and it has immensely increased our knowledge of pain processing.

There are several neuroimaging methods typically used to investigate the neural mechanisms involved in pain processing, including functional magnetic resonance imaging (FMRI), positron emission tomography (PET), magnetoencephalography (MEG) and electroencephalography (EEG). There are also other techniques that measure structural or metabolic changes in the brain such as diffusion tensor imaging, morphometric imaging and spectroscopy.

The most popular technique used for studying pain-related brain activation is FMRI. FMRI provides information about neural activity within the whole brain with anatomical accuracy and is completely non-invasive. This technique does not require exogenous contrast agents or radioligands as it uses blood properties to create image contrast. Although this is not a direct measure of brain activation, it has been demonstrated that FMRI signal correlates well with neuronal activity (Logothetis, 2003). There are two FMRI methods commonly used: blood oxygenation level dependent (BOLD) and arterial spin labeling (ASL) (Raichle and Mintun, 2006; Tracey and Mantyh, 2007).

BOLD FMRI measures local changes in blood oxygen content that normally take place in an active part of the brain as a result of an increased delivery of oxygen in response to an increase in 
metabolism. This creates the BOLD contrast. This method is not an absolute measure as it measures signal change relative to baseline. BOLD FMRI is typically used for studying brain activity in response to evoked brief stimuli and cannot be reliably used to study ongoing pain because of the need for a baseline and limits to stimulus duration.

ASL uses magnetically labeled blood as a contrast. It measures brain perfusion within the whole brain. Unlike BOLD FMRI, ASL FMRI can give absolute values of blood perfusion in $\mathrm{ml}$ of blood per $100 \mathrm{~g}$ of brain tissue per minute. Moreover, it does not require a change in stimulus and can be used for imaging activation lasting minutes. Therefore, it is suitable for studying the neural mechanisms of tonic evoked pain or clinical ongoing pain (Buxton, 2005; Tracey and Johns, 2010).

\section{Brain regions involved in pain perception}

The pathways and brain regions involved in pain processing have been relatively well-characterised using electrophysiological methods, histological tracers or lesion studies (Guilbaud et al., 2005). Neuroimaging provides an insight into the neurophysiological basis of pain not previously possible with other methods.

Neuroimaging established the role of the brain in pain by demonstrating extensively that the central nervous system is crucial 
for creating pain perception. It is important to remember that there is no single brain region that encodes "pain" and there are several regions that show somatotopy for pain (Baumgartner et al., 2010; Bingel et al., 2004). What becomes apparent is that there is no "primary pain cortex" for pain, an equivalent of the "primary sensory cortex" for touch (Tracey, 2011). There is no single region that, when dissected, will cause the pain to stop, although we know that lesion to the insular cortex may alter the pain sensation (Greenspan et al., 1999). There is probably not even a "primary nociceptive cortex". There are several regions however, including the posterior part of the insular cortex, the operculum or the secondary somatosensory cortex, that are possible candidates (Baumgartner et al., 2010; Tracey, 2011). The posterior insular cortex is particularly interesting as it encodes stimulus intensity across different modalities of pain and is only activated when an actual noxious input is present (Tracey, 2011).

As pain is a complex and multidimensional experience, it is represented by several regions within the brain (Apkarian et al., 2005; Tracey and Mantyh, 2007). Painful stimulation typically results in bilateral activation in the primary and secondary somatosensory cortices, the thalamus, the insular cortex and the anterior cingulate cortex. Activation in these regions is thought to be related to the sensory-discriminative aspects of pain processing, while the prefrontal and parietal cortices appear to participate in the cognitive aspect of pain and paradigms involving threat or anxiety usually 
evoke responses in the hippocampus and amygdala (Peyron et al., 2000; Tracey and Mantyh, 2007). It is important to emphasise the fact that there is no fixed set of regions responding to pain, there is no definitive "pain matrix" that is always present during pain perception. In addition, the pain-related regions are also not unique to pain experience (Tracey and Johns, 2010). Brain structures that are involved in pain processing are also engaged in processing and modulation of other sensory modalities as well as other emotional or cognitive states. As pain is multidimensional, its processing involves regions encoding not only the sensory but also the emotional, cognitive and motivational aspects of pain.

\section{Brain regions encoding intensity of pain}

Quantifying pain poses a challenge, as there is no objective measure of pain. Behavioural measures that are commonly used for characterising pain have several limitations as the patient is being asked to give a single number on a uni-dimensional scale to describe a complex experience of pain. In addition, the report is usually verbal which may be another limiting factor.

It is important to keep in mind that report bias is not just an artefact but an effect of pain modulation by internal, i.e., cognitive, emotional, genetic, and external factors such as stimulus properties (Chizh et al., 2009; Tracey and Mantyh, 2007). 
The perceived and reported pain is often not linearly related to the strength of the external input. There is a complex relationship between the nociceptive input and the pain experience resulting from it. In addition to that, nociception is not necessary to evoke pain perception, i.e., patients may feel pain without any external input for example in post stroke pain.

Neuroimaging offers an objective way of investigating pain by observing changes in brain activity related to pain. Brain imaging studies have demonstrated that the magnitude of pain-related activation is related to the intensity of the input and perceived pain (Coghill et al., 1999; Tracey and Mantyh, 2007)

As mentioned, the operculo-insular region is crucial for pain perception (Coghill et al., 1999); however, pain representation in that region is complex and there are several somatotopic representations of pain in that region (Baumgartner et al., 2010). The sensorydiscriminative aspect of the painful stimulation is also encoded in the primary and secondary somatosensory cortices, and the anterior cingulate cortex (Coghill et al., 1999) as well as in the thalamus, putamen and cerebellum (Bingel et al., 2002). The primary somatosensory cortex and the anterior cingulate cortex activation reflect specifically the intensity of pain experience rather than the input strength (Lee et al., 2008). On the other hand, information about stimulus intensity is encoded in the operculum, the insular cortex as well as the secondary somatosensory cortex; therefore, it may not be specific to pain (Baumgartner et al., 2010). In fact, 
stimulus intensity processing engages many cortical and subcortical regions, not only those encoding the sensory aspect of pain but also regions involved in attention, emotions and motor control (Coghill et al., 1999). This demonstrates that intensity is also relevant for other processes. The fact that intensity encoding is preserved across different brain regions supports the hypothesis of parallel pain processing (Coghill et al., 1999).

\section{Pain processing in terms of functional connectivity}

Perception is a dynamic process that is constantly modulated in a top-down fashion by attention and expectations. Top down influence means that even the earliest stages of cortical sensory processing are influenced by the higher cognitive processes (Gilbert and Sigman, 2007). Therefore, perception is better reflected by networks, than by brain regions in isolation. In order to understand the mechanisms involved in pain perception we have to study not just these regions, but connections between those regions and with other networks (Ploner et al., 2011).

Neuroimaging studies have demonstrated that the functional connectivity between the pain-related regions and other brain networks determines the subsequent pain experience (Ploner et al., 2010; Tracey and Johns, 2010). 
Functional connectivity tells us about changes in the strength of network connections between brain structures (Cauda et al., 2009). Those dynamic interactions between the cortical and subcortical structures are crucial for pain perception and modulation (Bingel et al., 2006; Valet et al., 2004). Moreover, the connectivity preceding the stimulus determines pain perception (Ploner et al., 2010). Ploner and colleagues demonstrated that the connectivity between the insular cortex and the brainstem predicts whether a potentially noxious stimulus will be perceived as painful (Ploner et al., 2010). This means that expectations bias perception (Keltner et al., 2006). In other words, the neural processing of the stimulus depends on the prior knowledge of the stimulus and the adequate adjustment preceding it. Neuroimaging studies have demonstrated that expectation of pain is associated with activation of pain-related regions such as the primary somatosensory cortex, the anterior cingulate cortex and the insular cortex (N.B. this means that there is activation within the pain-related regions in the absence of actual noxious stimulus) (Fairhurst et al., 2007; Ploghaus et al., 1999). The anterior insular cortex plays a key role in adjustment during the anticipation period and is involved in subsequent changes in pain processing (Ploner et al., 2010). It integrates information about the salience and potential threat of the upcoming stimulus. Moreover, the pre-stimulus activation in the anterior insular cortex results in a potentially painful stimulus (close to pain threshold) being perceived as actually painful (Wiech et al., 2010). The activation in the medial 
prefrontal cortex and the anterior cingulate cortex during the anticipatory period may inhibit the nociceptive transmission through cognitive appraisal, i.e., evaluation of meaning of the emotional stimuli (Kalisch et al., 2006; Lorenz et al., 2003).

\section{Pain modulation by attention and mood}

Neuroimaging has greatly advanced our knowledge of mechanisms involved in this modulation as it allowed us to use flexible paradigms to study different factors influencing pain experience (Tracey and Mantyh, 2007; Wiech et al., 2008).

Several neuroimaging studies have demonstrated that distraction changes the sensory aspect of pain and reduces pain ratings. These studies demonstrated that distraction is associated with a decrease in activity in the pain-related regions such as the anterior cingulate cortex, the thalamus and the insular cortex as well as with an increase of activity in the pain-modulatory regions, such as the anterior cingulate and the prefrontal cortex (Bantick et al., 2002).

Distraction was also associated with changes in networks and strength of the functional coupling between the anterior cingulate cortex and the prefrontal cortex as well as the periaqueductal grey (PAG) and posterior thalamus (Valet et al., 2004). An increase in activation in the periaqueductal grey predicted changes in reported pain intensity (Tracey et al., 2002). This suggests that, during distraction from pain, the anterior cingulate cortex and the prefrontal 
cortex exert a top-down influence on the thalamus and brainstem (Valet et al., 2004). The effect of distraction is most likely mediated via the descending pain modulatory system by inhibiting the nociceptive processing at the level of the dorsal horn (Tracey and Mantyh, 2007).

Depressed mood also increased the perceived pain intensity. Berna and colleagues showed that experimentally induced sad mood may alter pain perception in healthy people via the emotional regulatory networks (Berna et al., 2010). Sad mood resulted in stronger brain activation in response to pain in the regions processing pain and negative emotions, such as the anterior cingulate cortex and the hippocampus. There was also an increase in the activation in the prefrontal cortex related to appraisal (Berna et al., 2010).

In a recent experiment, Ploner and colleagues (Ploner et al., 2011) demonstrated that attention and emotions both modulate pain but through different mechanisms and they are related to different patterns of functional connectivity. Both attending to the stimulus and negative mood augmented pain and were associated with higher pain ratings. The magnitude of pain ratings correlated with activation of the anterior insular cortex. Interestingly, the attentional modulation altered the functional connectivity between the anterior insular cortex and frontoparietal regions whereas the emotional modulation changed the connectivity between the anterior insular cortex and limbic structures such as parahippocampal gyrus and amygdala. This suggests that the contextual modulation of pain depends on flexible 
functional interactions between the anterior insular cortex and the frontoparietal or the mediotemporal. Moreover, the modulation converged on a common structure, i.e., the anterior insular cortex. This suggests that the anterior insular cortex is important for integration of the sensory input and emotional and cognitive factors for the higher order representation of a state. This is in line with a study by Wiech and colleagues who demonstrated that the anterior insular cortex encodes stimulus salience thereby altering pain perception (Wiech et al., 2010). In a recent review, Wiech (Wiech et al., 2008) suggested that the descending modulatory system might interact or overlap with a more general system for attentional control that is not specific for pain.

\section{Placebo and nocebo modulation}

Neuroimaging studies on the placebo and nocebo effects are an excellent example demonstrating the complexity of pain processing and the mechanisms involved in modulating pain.

It is accepted that placebo is not a response/report bias (Hrobjartsson and Gotzsche, 2001). It is a result of interactions between the psychological and physiological effects of therapeutic procedures even in the absence of pharmacologically active analgesic substances. The placebo/nocebo effect has both unconscious components (conditioning) as well as conscious components (cognition, expectation, belief) (Bingel et al., 2011). 
Several studies (Wager et al., 2004) used neuroimaging to investigate the neural mechanisms of placebo/nocebo effect. Placebo manipulation was associated with a decrease of activation in pain-related regions (Bingel et al., 2006; Bingel et al., 2011), whereas nocebo effect was related to an increase of pain-related activity (Bingel et al., 2011). The magnitude of the activation change correlated with the change in the reported pain intensity which confirmed that placebo is a real effect not just a report bias (Bingel et al., 2011).

Neuroimaging studies demonstrated that placebo response is complex and may act via several mechanisms (Wiech et al., 2008) including: expectations about pain (Wager et al., 2004), cognitive appraisal (Petrovic et al., 2002), attentional control or the descending modulatory system including the opioid-related modulation and/or inhibition of afferent transmission (Wiech et al., 2008).

Placebo alters pain experience through modulation of expectation as has been demonstrated by Wager and colleagues (Wager et al., 2004) who showed an increased activation in the prefrontal cortex during anticipation of pain. The magnitude of the placebo effect correlated with the activation during the anticipation period in the orbitofrontal cortex, the dorsolateral prefrontal cortex and the rostral anterior cingulate cortex (Wager et al., 2004). This suggested that the rostral anterior cingulate cortex and the prefrontal cortex were involved in cognitive (expectation) control of pain processing related to the placebo effect and pain relief (Wager et al., 2004). The 
functional connectivity between the rostral anterior cingulate cortex and the prefrontal cortex is directly associated with a magnitude of pain relief related to the placebo effect (Petrovic et al., 2005; Wager et al., 2004). They also showed that the strongest placebo responders show the strongest coupling between the rostral anterior cingulate cortex and the prefrontal cortex (Petrovic et al., 2005) . The prefrontal cortex activity was associated with a reduction of pain (Lorenz et al., 2003; Seifert et al., 2009) probably via the top-down modulation of pain processing (Petrovic et al., 2010) related to cognitive appraisal of pain (Wiech et al., 2008). In addition, the orbitofrontal cortex is involved in expectations and attributing a specific value to external stimuli (Petrovic et al., 2005). From neuroimaging studies (Petrovic et al., 2005) we know that both emotional placebo and placebo analgesia act through the rostral anterior cingulate cortex and the orbitofrontal cortex. Ventro-orbital region was involved in top-down cognitive modulatory processes that might not be pain specific (Petrovic et al., 2002). This reflects a complex modulatory process that is not specific to placebo analgesia.

During the anticipation period, the activation in the prefrontal cortex and the anterior cingulate cortex correlated with the activation in the brainstem supporting the idea that prefrontal mechanisms are involved in top-down modulation of pain and the pain modulatory system within the brainstem for placebo and opioid analgesia (Petrovic et al., 2002; Wager et al., 2004). The descending modulatory system is crucial for the cognitive and psychological 
control of pain (Wiech et al., 2008). Wiech suggested that the descending modulatory pathway may be the final common pathway for analgesia or hyperalgesia (Wiech et al., 2008).

Bingel and colleagues (Bingel et al., 2011) used neuroimaging to investigate the neural mechanisms by which expectations, both positive and negative, interact with the physiological effects of an active drug (remifentanil). Behaviorally, placebo augmented the effect of the active drug whereas nocebo cancelled the analgesic effect. This was associated with a decrease and an increase of painrelated activation, respectively. Changes in pain rating (despite constant stimulus strength) were predicted by changes of activation in the rostral anterior cingulate cortex, i.e., increased activity during placebo and deactivated during the nocebo condition. As it has been mentioned earlier, the rostral anterior cingulate cortex plays an important role in placebo and opioid analgesia (Petrovic et al., 2005). In contrast, the hippocampus was active only during nocebo and was associated with increased anxiety ratings suggesting that it may be related to an impairment of analgesia due to negative expectations. This study demonstrated that expectation about the effect of the drug engaged mechanisms responsible for descending modulation of pain.

Several studies demonstrated that placebo analgesia engages the endogenous pain modulatory opioid network and leads to decreased afferent input to the brain (Petrovic et al., 2002; Wager et al., 2004). However, this is not the only mechanism and the placebo effect is 
complex and dynamic (Petrovic et al., 2010).

Neuroimaging was used to investigate whether placebo acts early through expectation, or late through opioid release, in response to pain or cognitive reappraisal of pain. Wager and colleagues examined temporal changes related to the placebo effect and observed an early change in the rostral anterior cingulate cortex suggesting that it is related to expectations. Other placebo-related changes were observed late suggesting that they may be associated with cognitive appraisal or opioid release in response to pain (Wager et al., 2004).

It has also been demonstrated that placebo analgesia shows significant differences from opioid analgesia despite involvement of opioidergic system in both. In the opioid condition, expectation for analgesia was met and was congruent with the level of nociceptive processing. In the placebo condition, the expectation was incongruent with the processing level of the nociceptive input (Petrovic et al., 2010).

In this study, the opioid rich rostral anterior cingulate cortex was more active during the opioid condition than the placebo condition. In contrast, the orbitofrontal cortex and the prefrontal cortex were more activated during the placebo condition probably reflecting the cognitive regulation of pain. Also the functional connectivity between rostral anterior cingulate cortex and prefrontal cortex was stronger in the placebo condition than during the opioid condition and the degree 
of connectivity was correlated with the degree of the placebo response. They interpreted this in terms of expectations and signal error. This supports the hypothesis that perception is a result of external incoming signals as well as the internal expectations. (Petrovic et al., 2010).

\section{Visualising ongoing pain}

The majority of functional imaging studies used BOLD FMRI and brief noxious stimuli; therefore, they were limited to studying acute pain. Acute pain is not necessarily the same as tonic pain.

Understanding the mechanisms involved in tonic pain is important for studies of clinical ongoing pain.

ASL offers a tool to examine clinical pain and the dynamics of pain processing during tonic or ongoing pain and subsequently improve understanding of pain processing and neural mechanisms involved in clinical pain.

Howard and colleagues (Howard et al., 2011) used ASL to study actual clinical pain, i.e., post-operative pain associated with tissue damage. They demonstrated that ongoing pain was associated with an increase of activation bilaterally in the pain-related regions and the magnitude of activation correlated with pain ratings. The painrelated activation was stable during ongoing pain and was reproducible between sessions. 
Owen and colleagues (Owen et al., 2010) used ASL to study a tonic muscular pain experimental model (intramuscular injection of hypertonic saline) in healthy volunteers. They investigated the temporal changes in the pattern of activation, i.e., the dynamics of pain evolution as the pain evolved from the first sharp acute pain to tonic pain. The transition from acute to tonic pain was associated with a decrease in the number of regions activated as well as a decrease in the spatial extent of the activation mostly in the brain regions involved in the processing of affective and cognitive dimensions of pain. Different rates of activity changed over time suggesting that different aspects of pain might evolve differently during ongoing pain. The insular cortex was the only region that showed a sustained activation during the tonic pain and activation in that region correlated strongly with pain intensity ratings. This study showed that ASL can be used to study ongoing pain processing and provide additional information about the specific mechanisms involved in this process.

\section{Future directions}

The big question for neuroimaging in pain research is whether these methods can be used as a read-out of pain experience independent of behavioural ratings. Neuroimaging has the potential to become an objective measure of pain and replace subjective report. Using neuroimaging to characterise pain without a need to rely on 
subjective pain ratings or descriptions of pain would be also of great benefit for measuring treatment efficacy.

Neuroimaging studies have shown that every individual has a unique pain signature, as a result of emotional and cognitive state, pain memories and pain beliefs, genetic profile, hormones and other factors that affect pain processing. Therefore, neuroimaging can potentially be used to phenotype pain (Tracey, 2011).

\section{Conclusions}

Neuroimaging changed the field of pain research. It made it possible to visualise pain processing and modulation in the brain in a noninvasive and non-interfering way. Therefore, it greatly increased our knowledge of pain processing at the level above the spinal cord.

Brain imaging gives an insight into the complexity of pain. Pain experience is an outcome of the external input as well as psychological, physiological and cognitive factors, which make pain very subjective. Therefore the "cerebral pain signature" is unique for a particular person at that particular moment.

Neuroimaging also provided complementary information about pain such as the effects of psychological, cognitive or pharmacological manipulations or the temporal dynamics of pain processing. Finally, brain imaging studies demonstrated that pain is a complex perception reflected by dynamic and flexible network interactions. 


\section{References}

Apkarian, A.V., Bushnell, M.C., Treede, R.D., and Zubieta, J.K. (2005). Human brain mechanisms of pain perception and regulation in health and disease. Eur J Pain 9, 463-484.

Bantick, S.J., Wise, R.G., Ploghaus, A., Clare, S., Smith, S.M., and Tracey, I. (2002). Imaging how attention modulates pain in humans using functional MRI. Brain 125, 310-319.

Baumgartner, U., Iannetti, G.D., Zambreanu, L., Stoeter, P., Treede, R.-D., and Tracey, I. (2010). Multiple Somatotopic Representations of Heat and Mechanical Pain in the Operculo-Insular Cortex: A High-Resolution fMRI Study. Journal of Neurophysiology 104, 2863-2872.

Berna, C., Leknes, S., Holmes, E.A., Edwards, R.R., Goodwin, G.M., and Tracey, I. (2010). Induction of Depressed Mood Disrupts Emotion Regulation Neurocircuitry and Enhances Pain Unpleasantness.

Biological Psychiatry 67, 1083-1090.

Bingel, U., Gaelscher, J., Weiller, C., and Bchel, C. (2004). Somatotopic representation of nociceptive information in the putamen: an eventrelated fMRI study. Cereb Cortex 14, 1340--1345.

Bingel, U., Lorenz, J., Schoell, E., Weiller, C., and Buchel, C. (2006).

Mechanisms of placebo analgesia: rACC recruitment of a subcortical antinociceptive network. Pain 120, 8-15.

Bingel, U., M. Quante, M., Knab, R., Bromm, B., Weiller, C., and Buechel, C. (2002). Subcortical structures involved in pain processing: evidence from single-trial fMRI. Pain 99, 313--321.

Bingel, U., Wanigasekera, V., Wiech, K., Ni Mhuircheartaigh, R., Lee, M.C., Ploner, M., and Tracey, I. (2011). The Effect of Treatment Expectation on Drug Efficacy: Imaging the Analgesic Benefit of the Opioid Remifentanil. Science Translational Medicine 3,70ra14.

Buxton, R.B. (2005). Quantifying CBF with arterial spin labeling. Journal of Magnetic Resonance Imaging 22, 723-726.

Cauda, F., Sacco, K., Duca, S., Cocito, D., D'Agata, F., Geminiani, G.C., and Canavero, S. (2009). Altered resting state in diabetic neuropathic pain. PLoS ONE 4, e4542.

Chizh, B.A., Priestley, T., Rowbotham, M., and Schaffler, K. (2009).

Predicting therapeutic efficacy -- Experimental pain in human subjects.

Brain Research Reviews 60, 243-254.

Coghill, R.C., Sang, C.N., Maisog, J.M., and Iadarola, M.J. (1999). Pain intensity processing within the human brain: a bilateral, distributed mechanism. J Neurophysiol 82, 1934-1943.

Fairhurst, M., Wiech, K., Dunckley, P., and Tracey, I. (2007). Anticipatory brainstem activity predicts neural processing of pain in humans. Pain $128,101--110$. 
Gilbert, C.D., and Sigman, M. (2007). Brain States: Top-Down Influences in Sensory Processing. Neuron 54, 677-696.

Greenspan, J.D., Lee, R.R., and Lenz, F.A. (1999). Pain sensitivity alterations as a function of lesion location in the parasylvian cortex. Pain 81, 273-282.

Guilbaud, G., Bernard, J.F., and Besson, J.M. (2005). Brain areas involved in nociception and pain. In Wall and Melzack's Textbook of Pain, S.

Koltzenburg, and M. McMahon, eds. (Churchill Livingstone).

Howard, M., Krause, K., Khawaja, N., Massat, N., Zelaya, F., Schumann, G., Huggins, J., Vennart, W., Williams, S., and TF, R. (2011). Beyond patient reported pain: perfusion magnetic resonance imaging demonstrates reproducible cerebral representation of ongoing post-surgical pain. PLoS One 6.

Hrobjartsson, A., and Gotzsche, P.C. (2001). Is the Placebo Powerless? New England Journal of Medicine 344, 1594-1602.

Kalisch, R.a.W., K., Critchley, H.D., and Dolan, R.J. (2006). Levels of appraisal: a medial prefrontal role in high-level appraisal of emotional material. Neuroimage 30, 1458--1466.

Keltner, J.R., Furst, A., Fan, C., Redfern, R., Inglis, B., and Fields, H.L. (2006). Isolating the Modulatory Effect of Expectation on Pain Transmission: A Functional Magnetic Resonance Imaging Study. J. Neurosci. 26, 4437-4443.

Lee, M.C., Zambreanu, L., Menon, D.K., and Tracey, I. (2008). Identifying brain activity specifically related to the maintenance and perceptual consequence of central sensitization in humans. J Neurosci 28, 11642-11649.

Logothetis, N.K. (2003). The underpinnings of the BOLD functional magnetic resonance imaging signal. J Neurosci 23, 3963--3971.

Lorenz, J., Minoshima, S., and Casey, K.L. (2003). Keeping pain out of mind: the role of the dorsolateral prefrontal cortex in pain modulation. Brain 126, 1079 - 1091.

Owen, D.G., Clarke, C.F., Ganapathy, S., Prato, F.S., and St. Lawrence, K.S. (2010). Using perfusion MRI to measure the dynamic changes in neural activation associated with tonic muscular pain. Pain 148, 375-386.

Petrovic, P., Dietrich, T., Fransson, P., Andersson, J., Carlsson, K., and Ingvar, M. (2005). Placebo in emotional processing--induced expectations of anxiety relief activate a generalized modulatory network. Neuron 46, 957-969.

Petrovic, P., Kalso, E., Petersson, K.M., Andersson, J., Fransson, P., and Ingvar, M. (2010). A prefrontal non-opioid mechanism in placebo analgesia. Pain 150, 59-65.

Petrovic, P., Kalso, E., Petersson, K.M., and Ingvar, M. (2002). Placebo and Opioid Analgesia-- Imaging a Shared Neuronal Network. Science 295, 1737-1740.

Peyron, R., Laurent, B., and Garcia-Larrea, L. (2000). Functional imaging of brain responses to pain. A review and meta-analysis (2000).

Neurophysiol Clin 30, 263-288. 
Ploghaus, A., Tracey, I., Gati, J.S., Clare, S., Menon, R.S., Matthews, P.M., and Rawlins, J.N. (1999). Dissociating pain from its anticipation in the human brain. Science 284, 1979-1981.

Ploner, M., Lee, M.C., Wiech, K., Bingel, U., and Tracey, I. (2010). Prestimulus functional connectivity determines pain perception in humans. Proceedings of the National Academy of Sciences 107, 355360.

Ploner, M., Lee, M.C., Wiech, K., Bingel, U., and Tracey, I. (2011). Flexible Cerebral Connectivity Patterns Subserve Contextual Modulations of Pain. Cerebral Cortex 21, 719-726.

Raichle, M.E., and Mintun, M.A. (2006). Brain work and brain imaging. Annu Rev Neurosci 29, 449-476.

Seifert, F., Bschorer, K., De Col, R., Filitz, J., Peltz, E., Koppert, W., and Maihoefner, C. (2009). Medial Prefrontal Cortex Activity Is Predictive for Hyperalgesia and Pharmacological Antihyperalgesia. The Journal of Neuroscience 29, 6167-6175.

Tracey, I. (2011). Can neuroimaging studies identify pain

endophenotypes in humans? Nature Reviews Neurology 7, 173-181. Tracey, I., and Johns, E. (2010). The pain matrix: reloaded or reborn as we image tonic pain using arterial spin labelling. Pain 148, 359-360. Tracey, I., and Mantyh, P.W. (2007). The cerebral signature for pain perception and its modulation. Neuron 55, 377--391.

Tracey, I., Ploghaus, A., Gati, J.S., Clare, S., Smith, S., Menon, R.S., and Matthews, P.M. (2002). Imaging attentional modulation of pain in the periaqueductal gray in humans. J Neurosci 22, 2748-2752.

Valet, M., Sprenger, T., Boecker, H., Willoch, F., Rummeny, E., Conrad, B., Erhard, P., and Tolle, T.R. (2004). Distraction modulates connectivity of the cingulo-frontal cortex and the midbrain during pain--an fMRI analysis. Pain 109, 399-408.

Wager, T.D., Rilling, J.K., Smith, E.E., Sokolik, A., Casey, K.L., Davidson, R.J., Kosslyn, S.M., Rose, R.M., and Cohen, J.D. (2004). Placebo-Induced Changes in fMRI in the Anticipation and Experience of Pain. Science 303, 1162-1167.

Wiech, K., Lin, C.-S., Brodersen, K.H., Bingel, U., Ploner, M., and Tracey, I. (2010). Anterior Insula Integrates Information about Salience into Perceptual Decisions about Pain. The Journal of Neuroscience 30, 16324-16331.

Wiech, K., Ploner, M., and Tracey, I. (2008). Neurocognitive aspects of pain perception. Trends Cogn Sci 12, 306--313. 